\title{
EL MÉTODO DE DIFERENCIAS FINITAS EN EVALUACIÓN DE OPCIONES REALES ${ }^{1}$ THE FINITE DIFFERENCE METHOD IN REAL OPTIONS VALUATION
}

\author{
Sebastián Otero G. ${ }^{2} \quad$ Alejandro Andalaft C. ${ }^{2} \quad$ Evelyn Vásquez S. ${ }^{2}$ \\ Recibido el 27 de diciembre de 2006, aceptado el 29 de octubre de 2007 \\ Received: December 27, 2006 Accepted: October 29, 2007
}

\begin{abstract}
RESUMEN
Una respuesta a las falencias de los métodos tradicionales de evaluación de proyectos de inversión corresponde a la metodología de "Opciones Reales" que proviene del análisis de opciones sobre instrumentos financieros desarrollado en la década de los 70. Esta técnica, aplicada inicialmente a activos no financieros o reales, ha sido aplicada con singular éxito en la evaluación de proyectos de inversión y se presenta como una alternativa a los métodos de evaluación tradicionales, pues incluye tanto los aspectos estratégicos como de la teoría financiera. La variación fundamental que experimenta la evaluación de proyectos de inversión mediante opciones reales con respecto a la realizada mediante los métodos tradicionales es la incorporación de la incertidumbre y la flexibilidad como elementos que agregan valor al proyecto.

En este trabajo se realiza un recorrido del estado del arte en lo que respecta a la teoría de opciones reales para la evaluación de proyectos de inversión mediante diferencias finitas. Se describe la importancia de los componentes económicos y estratégicos a la hora de evaluar proyectos de inversión ante escenarios de incertidumbre, analizando las características de esta metodología, destacando sus virtudes y defectos e identificándose su aplicabilidad en la evaluación de proyectos de inversión.

Finalmente se realiza una implementación del método de diferencias finitas implícitas (DFI) para la evaluación de un caso real que cuenta con las opciones de esperar, abandonar, contraer, expandir y cambiar a mejor uso alternativo, evaluándose para todas las opciones y combinaciones de opciones posibles. Los resultados fueron comparados con la metodología tradicional $(V A N)$ y el árbol binomial con transformada logarítmica $(A B T L)$. Las dos metodologías $(D F I$ y $A B T L)$ entregan valores equivalentes, siendo ambos superiores a los obtenidos mediante la metodología tradicional (VAN), diferencia que representa su capacidad de incorporar en el análisis el valor de la flexibilidad operativa del proyecto.
\end{abstract}

Palabras clave: Opciones reales, diferencias finitas, flexibilidad, incertidumbre, evaluación de proyectos.

\begin{abstract}
In the past few years, real options, an extension of financial derivatives, have arisen as an alternative to traditional valuation methods, such as net present value (NPV). The key attribute of real options is that they take into consideration the uncertainty and flexibility involved in investment valuation. This article provides an overview of the finite difference method, by presenting an application to the real options valuation. The empirical section of the article, which makes use of the implicit finite difference method (IFD), analyzes the options of waiting, abandoning, contracting, expanding and switching, by valuing all the options involved and their possible combinations. The results are compared with those of the NPV method and the binomial tree with a logarithmic transformation (BTLT). Both methods (IFD and BTLT) yield similar results, being both greater than those provided by the NPV. This difference comes to no surprise as it represents the value of the flexibility associated to an investment opportunity.
\end{abstract}

Keywords: Real Options, finite differences, flexibility, uncertainty, project valuation.

\footnotetext{
1 Este trabajo se desarrolló en el marco de una Tesis de Magíster en Ingeniería Industrial y contó con el apoyo de la Dirección de Investigación de la Universidad de Concepción, a través del proyecto DIUC 205.097.008-1.

2 Departamento de Ingeniería Industrial. Facultad de Ingeniería. Universidad de Concepción. Concepción, Chile.

Email: sotero@udec.cl, aandalaft@udec.cl, evelynvasquez@udec.cl
} 


\section{INTRODUCCIÓN}

El valor de un proyecto se relaciona no sólo con los flujos de caja que produce, sino también con las oportunidades estratégicas que genera. Sin embargo, este fenómeno no puede ser detectado por los métodos tradicionales de evaluación de proyectos, los que generalmente llevan a tomar la decisión de no invertir en proyectos con alto valor estratégico, pero baja rentabilidad.

Al centrarse en la teoría financiera, se ve que los problemas básicos que poseen los métodos de flujo de caja descontado para realizar la evaluación económica de proyectos, se relacionan con el hecho de que son incapaces de capturar adecuadamente la flexibilidad operativa que se posee al momento de decidir, ignorando la capacidad de revisar o modificar las decisiones producto de la disipación de la incertidumbre producida por el paso del tiempo [22].

Los métodos de evaluación de instrumentos derivados han alcanzado un alto nivel de madurez. Sin embargo, los desafíos siguen apareciendo en diferentes frentes y es seguro que seguirá siendo un reto el desarrollar un modelo de valoración que se ajuste mejor a la información observada. La permanente evolución de la tecnología computacional expandirá las cantidades y tipo de información posibles de procesar, de la misma manera en que aumentará la cantidad de modelos financieros estudiados y solucionados numéricamente. Sin embargo, el desarrollo computacional por sí solo es de utilidad muy limitada. Muchos problemas son exponenciales en sus requerimientos de memoria y tiempo computacional, y esto requiere de avances en el desarrollo de algoritmos [9].

Los objetivos de este trabajo son conocer y aplicar la metodología de opciones reales para la evaluación de proyectos de inversión mediante diferencias finitas, contrastando los resultados obtenidos por esta metodología con los obtenidos por otros métodos tradicionales de valoración. Se pretende reafirmar la importancia del enfoque de opciones reales como herramienta de evaluación de proyectos de inversión, aportando así un nuevo eslabón a la cadena de investigación en esta área de las finanzas modernas.

Si bien es cierto que la incertidumbre no es importante en inversiones para las cuales los precios relevantes sean razonablemente predecibles, la volatilidad cobra una importancia vital en aquellos proyectos donde las fluctuaciones de precios del orden del 30\% anual son muy frecuentes. Bajo estas condiciones, la práctica del $V A N$ de reemplazar la distribución de los precios futuros por sus valores esperados tiende a causar errores en el cálculo de los flujos de caja esperados, como también de las tasas de descuento apropiadas.

En segundo lugar, el VAN no considera las flexibilidades inherentes a un proyecto, tales como la opción de posponer inversiones, aumentar la producción en caso que los precios suban, reducirla en caso que éstos bajen, o bien, abandonar el proyecto si las condiciones son muy desfavorables.

En tercer lugar, la estimación de una tasa de descuento que refleje el riesgo de los flujos de caja a lo largo de toda la vida del proyecto resulta muy difícil, ya que la incertidumbre puede ser variable a lo largo del tiempo y además este único parámetro debe considerar el nivel de producción al cual opera el proyecto, al igual que sus distintas flexibilidades.

Finalmente, una ventaja del método de opciones reales es que además de evaluar el activo derivado real, también determina la política óptima que maximiza el valor de este proyecto. Estas estrategias están determinadas por valores críticos de las variables inciertas. Estos umbrales señalan cuándo es óptimo ejercer las distintas opciones de las que se dispone, por ejemplo, invertir, almacenar o cerrar [15].

\section{LA TEORÍA FINANCIERA Y EVALUACIÓN DE PROYECTOS}

\section{Orígenes de las Opciones Reales}

Una respuesta a las falencias de los métodos tradicionales corresponde a la metodología de Opciones Reales que se inicia con Stewart Myers, académico del Massachusetts Institute of Technology (MIT). Esta metodología proviene del análisis de opciones sobre instrumentos financieros desarrollado en la década de los '70. Esta técnica, aplicada inicialmente a activos no financieros o reales, ha sido desarrollada con singular éxito en la evaluación de proyectos de inversión y se presenta como una alternativa a los métodos de evaluación tradicionales, pues incluye tanto los aspectos estratégicos como de la teoría financiera.

La variación fundamental que experimenta la evaluación de proyectos de inversión mediante opciones reales con respecto a la realizada mediante los métodos tradicionales es la incorporación de la incertidumbre como elemento que agrega valor al proyecto [13].

Las opciones reales representan la flexibilidad del proyecto y son las herramientas de decisión que permiten hacer una revisión continua de las decisiones tomadas en torno al 
proyecto, permitiendo así no seguir un plan establecido y adaptarse a los eventos que el futuro depara [22].

El enfoque basado en las opciones reales no se reduce a usar un nuevo conjunto de ecuaciones y modelos de evaluación. Exige cambiar la forma de encuadrar las decisiones estratégicas. La pregunta deja de ser “¿Qué ganamos si nos movemos del punto $A$ al punto $B$ ?" para hacerse algo más compleja: "Si avanzamos del punto $A$ al punto $B$, ¿qué opciones se nos abrirán en el camino y qué ganaremos por el hecho de contar con ellas?". El primer paso para reorientar el pensamiento estratégico es, entonces, identificar las opciones reales que ofrecen las decisiones de inversión [3].

Las opciones reales incorporan el concepto de evaluación bajo la inexistencia de oportunidades de arbitraje y permiten lograr el alineamiento entre las decisiones estratégicas de inversión y los mercados financieros. Esto se realiza mediante la utilización de un activo subyacente transado en el mercado, con una rentabilidad esperada igual a la del proyecto, que represente a través de la evolución de su precio en el tiempo, el comportamiento de la variabilidad de los flujos de caja del proyecto durante el horizonte de evaluación [11].

\section{La flexibilidad}

El concepto de "opción" se asemeja bastante a la unidad básica de la flexibilidad. El concepto de opciones reales es un derecho, pero no una obligación, de realizar algo a un determinado costo dentro de un periodo específico de tiempo. Este concepto modela la flexibilidad como una estructura asimétrica de derecho y obligación para enfrentar un costo en un intervalo de tiempo. Corresponde a la estructura básica de las decisiones humanas: obtener ventaja de condiciones favorables y evitar condiciones de riesgo adversas [23].

Al analizar la flexibilidad de un proyecto se debe considerar la capacidad de modificar las decisiones estratégicas tomadas inicialmente, en respuesta a las eventualidades que puedan ocurrir en el futuro. Por lo mismo resulta fundamental incorporar la incertidumbre implícita en todo proyecto al realizar el análisis, determinando las distintas oportunidades de acción y decisión que puedan presentarse durante la vida útil del proyecto en la medida en que el escenario existente al evaluar el proyecto se vea modificado.

La metodología que utiliza la teoría de opciones reales elimina la incertidumbre asociada al activo derivado real a través de la construcción de un portafolio libre de riesgo entre el activo subyacente y el activo derivado que se quiere evaluar. Además, tal construcción se basa en la ausencia de oportunidades de arbitraje con lo cual el retorno del portafolio, que está formado por instrumentos que tienen retornos perfectamente correlacionados, debe ser igual a la tasa de interés libre de riesgo. Por otro lado, la evaluación mediante el enfoque de opciones reales utiliza información de los mercados para conocer los precios de los activos subyacentes, lo que permite evitar la estimación de parámetros.

Por último, una de las ventajas más importantes de esta metodología es que proporciona una política óptima de operación que maximiza el valor del activo real. Tal política se caracteriza por entregar un conjunto de valores críticos de las variables inciertas presentes en un proyecto, que indica cuándo y en qué instante es óptimo ejercitar la opción [16].

Básicamente, el enfoque utilizado en los modelos de valoración de opciones consiste en describir la incertidumbre asociada a los flujos de caja de un proyecto a través de la especificación de un proceso estocástico para una o más variables que describan la evolución en el tiempo de su valor, y en suponer que existe en el mercado un portafolio de activos que hace posible replicar el comportamiento estocástico del proyecto, teniendo perfecta correlación con él, de modo de eliminar dinámicamente todo el riesgo. De este modo, aplicando el concepto de ausencia de oportunidades de arbitraje, el valor del proyecto será equivalente al valor del portafolio replicador, y este podrá ser determinado resolviendo un sistema de ecuaciones diferenciales parciales que representan la evolución estocástica del portafolio replicador [13].

Según Black y Scholes [7], el retorno de las opciones se encuentra perfectamente correlacionado con el retorno de sus activos subyacentes. Luego, la formación de un portafolio que contenga al activo subyacente y a la opción da origen a la ecuación diferencial que describe el precio de este activo derivado, en función de su activo subyacente, la que tendrá tantas dimensiones como variables de estado se utilicen en la evaluación.

\section{Tipos de opciones reales}

Los proyectos de inversión pueden ser analizados como una colección de opciones reales representadas como distintas combinaciones de opciones de compra y venta (put y call), según las oportunidades que existan durante la vida útil del proyecto. La valoración de este paquete de opciones puede ser realizada mediante sofisticadas técnicas financieras que consideran complejos métodos de resolución y que permiten reflejar la incertidumbre, permitiendo obtener una aproximación del valor actual del proyecto [13]. 
Así como el dueño de una opción financiera posee el derecho pero no la obligación de ejercer la compra o venta del activo, el dueño de una opción sobre los flujos de caja de un proyecto posee el derecho pero no la obligación de adquirir el valor presente de los flujos de caja del proyecto, o sea una opción real sobre un activo real.

Uno de los principales aportes de las opciones reales es el permitir que el poseedor de la oportunidad tome la decisión sobre el momento de la inversión, incorporando los conceptos de flexibilidad y aprendizaje. Así, puede surgir la opción de posponer, contraer o expandir una inversión para aprovechar al máximo una oportunidad, sin enfrentarse a un plan fijo a seguir, como lo proponen los métodos tradicionales de evaluación.

De lo anterior se puede concluir que el valor de un proyecto de inversión al considerar la flexibilidad operativa que entregan las opciones reales, puede ser calculado como la suma del valor actual neto del proyecto sin flexibilidad (el tradicional VAN) más el valor de la opción correspondiente, lo que se denomina "valor actual neto expandido", o sea:

$$
V A N_{\text {exp }}=V A N_{\text {trad }}+\text { premio por opción }
$$

En la gran mayoría de los casos, los proyectos de inversión presentan una serie de opciones dentro de su vida útil, por lo que el valor de éstos debe ser calculado incorporando los premios correspondientes a cada opción. Sin embargo, el valor de un conjunto de opciones no es igual a la suma de las opciones individuales que lo componen, ya que durante el horizonte de evaluación existirán interacciones entre las diversas opciones presentes. Por lo tanto, es necesario tomar en cuenta simultáneamente todas las opciones presentes en el proyecto para poder evaluar correctamente el valor del proyecto [22].

\section{La valoración neutral al riesgo}

En los inicios de la década de 1970, Fischer Black y Myron Scholes realizaron un importante aporte al derivar una ecuación que representa el comportamiento del precio de cualquier instrumento derivado dependiente de una acción que no pague dividendos y utilizaron esta ecuación para obtener el valor de diferentes opciones europeas de put y call sobre acciones.

Sin lugar a dudas la valoración neutral al riesgo es la herramienta más importante para el análisis de instrumentos derivados. Proviene de una de las propiedades clave de la ecuación diferencial de Black-Scholes y está referida a que la ecuación no incluye ninguna variable que tenga relación con la actitud al riesgo de los inversores. Las variables que aparecen en dicha ecuación son el precio actual de la acción, el tiempo, la volatilidad del precio de la acción y la tasa de interés libre de riesgo, todos independientes del nivel de aversión al riesgo, lo que permite utilizar el simple supuesto de que todos los inversores son neutrales al riesgo.

\section{LOS MÉTODOS NUMÉRICOS}

Durante el presente siglo, avanzados modelos matemáticos han sido ampliamente utilizados en muchas áreas, tales como la medicina, economía y ciencias sociales. Sin embargo, algunas aplicaciones de problemas matemáticos no pueden ser resueltas convenientemente a través de fórmulas exactas, razón por la cual se debe recurrir a ciertas restricciones que conllevan a casos especiales o a modelos simplificados que sí cuentan con soluciones analíticas. Cuando las aproximaciones no son suficientes, se hace necesario optar por una resolución que involucre gran cantidad de cálculos numéricos. Ante esta situación y con los enormes avances en el área de la computación, la aplicabilidad de los métodos numéricos se ha desarrollado notablemente.

Específicamente en el campo de las finanzas, ocurre que para valorizar un activo real usando la teoría de opciones reales, se debe resolver una ecuación diferencial parcial que tiene tantas dimensiones como variables de estado tenga el problema en cuestión. En las evaluaciones más complejas que incorporan múltiples opciones, generalmente no existe solución analítica, de forma tal que se hace necesaria la utilización de métodos numéricos [16].

En la teoría de opciones, los métodos numéricos que se aplican para resolver un problema de evaluación pueden clasificarse básicamente en dos tipos: El primero abarca a aquellos que aproximan directamente el proceso estocástico que sigue el activo subyacente. Dentro de éstos se incluyen la simulación de Montecarlo y algunas aproximaciones por malla, tales como los métodos de aproximación binomial y el de aproximación binomial con transformada logarítmica.

En la segunda clasificación se abarcan los métodos que aproximan la ecuación diferencial parcial que se obtiene. Tal es el caso de los métodos de integración numérica y los esquemas de diferencias finitas explícitas e implícitas [1]. 


\section{El método de diferencias finitas}

El método de diferencias finitas $(D F)$ para la evaluación de opciones fue propuesto inicialmente por Brennan y Schwartz [8]. Una de las grandes ventajas de este método corresponde a la riqueza en teoría, algoritmos y softwares numéricos existentes que pueden ser utilizados para enfrentar el problema. Temas como la consistencia numérica, convergencia y estabilidad han sido profundamente estudiados. La generalidad del método $D F$ es especialmente importante en modelos que se extienden más allá de los coeficientes constantes del modelo de Black-Scholes. El método $D F$ puede manejar procesos con coeficientes variables en el tiempo, modelos de tasa de interés simples o multifactoriales, etc. Además ofrece una considerable flexibilidad en las opciones de mallas en las dimensiones de tiempo y espacio, lo que resulta útil al tratar con dividendos discretos, barreras y otros escenarios comunes, mientras que a través de aproximaciones de mayor orden es posible mejorar la convergencia [9].

Los métodos $D F$ valorizan un derivado resolviendo la ecuación diferencial que es satisfecha por el derivado. La ecuación diferencial es convertida en una serie de ecuaciones diferenciales, las que son resueltas de manera iterativa.

Para ilustrar esta metodología, consideremos cómo se aplicaría para evaluar una opción put americana sobre una acción sin dividendos. La ecuación diferencial que la opción debe satisfacer es la misma presentada por Black y Scholes:

$$
\frac{\partial f}{\partial t}+r S \frac{\partial f}{\partial S}+\frac{1}{2} \sigma^{2} S^{2} \frac{\partial^{2} f}{\partial S^{2}}=r f
$$

Luego se divide el tiempo total, entre el instante cero y el instante de madurez de la opción, $T$, en una serie de intervalos iguales. Suponemos que $\Delta t=T / N$ y consideramos los siguientes $N+1$ intervalos:

$$
0, \Delta t, 2 \Delta t, 3 \Delta t \ldots T
$$

Así también, se elige una serie de precios de acción equidistantes, suponiendo que $S_{\max }$ es aquel precio de la acción suficientemente alto tal que si es alcanzado, la opción put virtualmente no tiene valor. Se define un $\Delta s=S_{\max } / M$ y consideramos un total de $M+1$ posibles precios de acción:

$$
0, \Delta S, 2 \Delta S, 3 \Delta S \ldots S_{\max }
$$

Se asume que uno de estos valores es el valor actual de la acción.

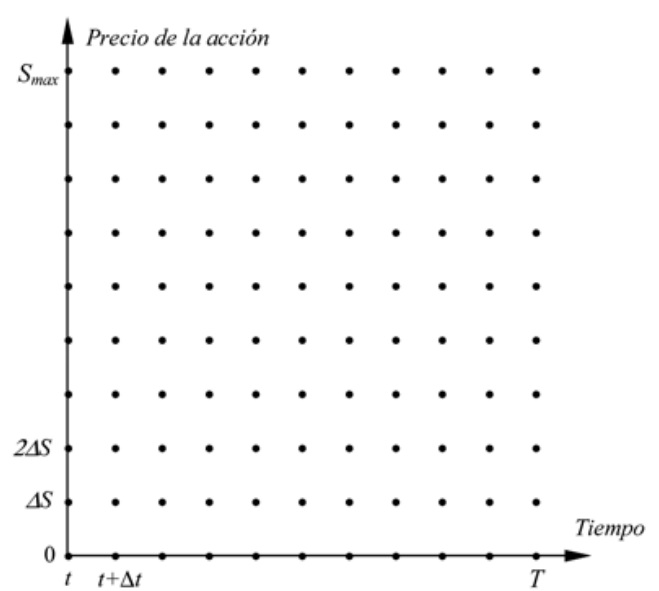

Figura 1. Malla de puntos para la aproximación mediante diferencias finitas.

Esta aproximación general es representada gráficamente en la figura 1, en la que se presenta una malla de $(M+1)$ por $(N+1)$ puntos. El punto $(i, j)$ de la malla es el punto que corresponde al tiempo $i \Delta t \mathrm{y}$ al precio de la acción $j \Delta S$. Vamos a utilizar la variable $f_{i, j}$ para denotar el valor de la opción en el punto $(i, j)[17]$.

\section{El método de las diferencias finitas implícitas}

Para un punto interior de la malla $(i, j), \partial f / \partial S, \partial f / \partial t, \partial^{2} f / \partial S^{2}$ pueden ser aproximados mediante $D F$, obteniéndose:

$$
\begin{aligned}
& \frac{f_{i+1, j}-f_{i, j}}{\Delta t}+r j \Delta S \frac{f_{i, j+1}-f_{i, j-1}}{2 \Delta S}+ \\
& \frac{1}{2} \sigma^{2} j^{2} \Delta S^{2} \frac{f_{i, j+1}+f_{i, j-1}-2 f_{i, j}}{\Delta S^{2}}=r f_{i, j}
\end{aligned}
$$

$\operatorname{para} j=1,2, \ldots, M-1$ e $i=0,1, \ldots, N-1$.

Reordenando términos, obtenemos:

$$
a_{j} f_{i, j-1}+b_{j} f_{i, j}+c_{j} f_{i, j+1}=f_{i+1, j}
$$

donde

$$
\begin{gathered}
a_{j}=\frac{1}{2} r j \Delta t-\frac{1}{2} \sigma^{2} j^{2} \Delta t \\
b_{j}=1+\sigma^{2} j^{2} \Delta t+r \Delta t \\
c_{j}=-\frac{1}{2} r j \Delta t-\frac{1}{2} \sigma^{2} j^{2} \Delta t
\end{gathered}
$$

El valor de la opción put en el tiempo $T$ es $\max \left[X-S_{T}, 0\right]$ donde $S_{T}$ es el valor de la acción en el tiempo $T$. Por lo tanto: 


$$
f_{N, j}=\max [X-j \Delta S, 0] \quad j=0,1, \ldots, M
$$

El valor de la opción put cuando el valor de la acción es cero será $X$, por lo tanto:

$$
f_{i, 0}=X \quad i=0,1, \ldots
$$

El valor de la opción put tiende a cero cuando el valor de la acción tiende a infinito, por lo tanto podemos utilizar la siguiente aproximación:

$$
f_{i, M}=0 \quad i=0,1, \ldots, 1
$$

Las ecuaciones (4), (5) y (6) definen los valores de la opción put a lo largo de tres de las aristas de la malla indicada en la figura (1), donde $S=0, S=S_{\text {max }}$ y $t=T$. Nos queda por utilizar la ecuación (3) para llegar al valor de $f$ para todo el resto de los puntos de la malla. Primero se calculan los valores correspondientes al tiempo $T-\Delta t$. La ecuación (3) con $i=N-1$ nos entrega $M-1$ ecuaciones simultáneas:

$$
a_{j} f_{N-1, j-1}+b_{j} f_{N-1, j}+c_{j} f_{N-1, j+1}=f_{N, j}
$$

para $j=1,2, \ldots, M-1$. Los lados derechos de las ecuaciones son conocidos a partir de (4). Además, de las ecuaciones (5) y (6) tenemos:

$$
\begin{aligned}
& f_{N-1,0}=X \\
& f_{N-1, M}=0
\end{aligned}
$$

Las ecuaciones (7) son $M-1$ ecuaciones las que pueden ser resueltas para así conocer las $M-1$ incógnitas: $f_{N-1,1}$, $f_{N-1,2}, \ldots, f_{N-1, M-1}$. Luego que se ha realizado esto, cada valor de los $f_{N-1, j}$ es comparado con $X-j \cdot \Delta S$. Si $f_{N-1, j}<X-j \cdot \Delta S$, será óptimo ejercer la opción en el tiempo $T-\Delta t$ y $f_{N-1, j}$ toma el valor de $X-j \cdot \Delta S$. A continuación, los nodos correspondientes al tiempo $T-2 \Delta t$ son calculados de la misma manera, y así para los otros nodos. Finalmente se obtienen $\operatorname{los} f_{0,1}, f_{0,2}, f_{0,3}, \ldots, f_{0, M-1}$. Uno de ellos será el valor de la opción de interés [17].

\section{APLICACIÓN DEL MÉTODO DE DIFERENCIAS FINITAS}

Para implementar la metodología de diferencias finitas a casos con múltiples opciones reales es necesario realizar un procedimiento de resolución paso a paso muy detallado de manera de tomar en cuenta todas las consideraciones de la metodología simultáneamente con las condiciones de borde, las opciones existentes en cada instante de tiempo y las diferentes combinaciones de opciones que se pueden producir.
El valor de un conjunto de opciones no es igual a la suma de las opciones individuales que lo componen, ya que durante la vida del proyecto existen interacciones entre las opciones presentes, cuya incidencia en el valor del proyecto no puede ser determinada a priori [14]. Es por esto que para aplicar la metodología $D F$ a un caso real de evaluación de un proyecto que contemple diversas opciones reales es necesario considerar todas las opciones simultáneamente y las diferentes combinaciones de opciones posibles de encontrar en el proyecto.

\section{Descripción del proceso de resolución}

Siguiendo con la metodología de resolución mediante $D F I$, el problema se basa principalmente en resolver el sistema de ecuaciones indicado anteriormente (ec. 3):

$$
a_{j} f_{i, j-1}+b_{j} f_{i, j}+c_{j} f_{i, j+1}=f_{i+1, j}
$$

En este sistema (11), $\operatorname{los} f_{N, j}$ iniciales (con $i=N-1$ ) son determinados a partir de la discretización de los valores y considerando la opción real presente al momento de expiración del proyecto, es decir, cuando $t=T$.

El sistema se puede descomponer para $j$ desde 1 hasta $M-1$ :

$$
\begin{aligned}
& j=1 \quad a_{1} f_{i, 0}+b_{1} f_{i, 1}+c_{1} f_{i, 2}=f_{i+1,1} \\
& j=2 \quad a_{2} f_{i, 1}+b_{2} f_{i, 2}+c_{2} f_{i, 3}=f_{i+1,2} \\
& \begin{array}{lllll} 
& \ldots & \ldots & \ldots & \ldots
\end{array} \\
& j=M-2 \quad a_{M-2} f_{i, M-3}+b_{M-2} f_{i, M-2}+c_{M-2} f_{i, M-1}=f_{i+1, M-2} \\
& j=M-1 \quad a_{M-1} f_{i, M-2}+b_{M-1} f_{i, M-1}+c_{M-1} f_{i, M}=f_{i+1, M-1}
\end{aligned}
$$

Las condiciones de borde 8 y 9 nos entregan los valores de $f_{i, 0}$ y $f_{i, M}$ respectivamente, las que se incorporan como ecuaciones al sistema para obtener un sistema de $M+1$ ecuaciones con $M+1$ incógnitas de tipo:

$$
A \times F_{N-1}=F_{N}
$$

en el que $A$ corresponde a una matriz "tri-diagonal" de dimensiones $(M+1) x(M+1)$ que incorpora las condiciones de borde y los parámetros $a_{j}, b_{j}$ y $c_{j}$ que acompañan a las incógnitas $f_{i, j}$ del sistema de ecuaciones. $F_{N-1}$ corresponde al vector de $M+1$ incógnitas y $F_{N}$ corresponde al vector de $M+1$ elementos conocidos. El sistema se resuelve mediante la inversión de la matriz tri-diagonal y premultiplicándola por el vector conocido de la forma:

$$
F_{N-1}=A^{-1} \times F_{N}
$$




\begin{tabular}{|c|c|c|c|c|c|c|c|c|}
\hline \multirow{2}{*}{\multicolumn{2}{|c|}{$\begin{array}{l}\text { Valor del flujo } \\
\text { de caja sin } \\
\text { opción } \\
S=\operatorname{Smax} \quad j=M\end{array}$}} & \multicolumn{6}{|c|}{$\begin{array}{l}\text { Valor de la opción para cada instante } t \\
\text { y para cada valor del flujo de caja } S\end{array}$} & \\
\hline & & $f_{O, M}$ & $f_{I, M}$ & $f_{2, M}$ & $\ldots$ & $f_{N-1, M}$ & $f_{N, M}$ & \\
\hline \multirow[t]{2}{*}{$S=(M-1) \Delta s$} & \multirow[t]{2}{*}{$j=M-1$} & $f_{0, M-1}$ & $f_{l, M-1}$ & $f_{2, M-1}$ & $\cdots$ & $f_{N-1, M-1}$ & $f_{N, M-1}$ & \\
\hline & & : & $\vdots$ & $\vdots$ & & $\vdots$ & & \\
\hline$S=2 \Delta s$ & $j=2$ & $f_{0,2}$ & $f_{I, 2}$ & $f_{2,2}$ & $\ldots$ & $f_{N-1,2}$ & $f_{N, 2}$ & \\
\hline \multirow{4}{*}{$\begin{array}{c}S=\Delta s \\
S=0\end{array}$} & \multirow{4}{*}{$\begin{array}{l}j=1 \\
j=0\end{array}$} & $f_{0,1}$ & $f_{1,1}$ & $f_{2,1}$ & $\ldots$ & $f_{N-l, I}$ & $f_{N, 1}$ & \\
\hline & & $f_{0,0}$ & $f_{1,0}$ & $f_{2,0}$ & $\ldots$ & $f_{N-1,0}$ & $f_{N, 0}$ & \\
\hline & & $i=0$ & $i=1$ & $I=2$ & & $i=N-1$ & $i=N$ & \\
\hline & & $t=0$ & $t=\Delta t$ & $t=2 \Delta t$ & & $t=T-\Delta t$ & $t=T$ & Tiempo \\
\hline
\end{tabular}

Figura 2. Matriz solución obtenida mediante diferencias finitas implícitas.

La matriz $A$ es también llamada matriz de transición, ya que está compuesta por las probabilidades de transición desde el estado $i$ en el momento $j$, al estado $i+1$ ó $i$ - 1 en el momento $j+1$, obtenida a partir de los coeficientes de la ecuación discretizada.

A partir de la columna $N$ (que almacena los valores de la opción en el tiempo $T$, o sea en la fecha de expiración) se encuentran los elementos de la columna $N$-1, que corresponderán a los valores de la opción para el tiempo $T-\Delta t$. Los valores encontrados son comparados con la opción existente en ese instante de tiempo (si es que existe alguna opción) para ver si es óptimo o no ejercer la opción en ese instante, y así sucesivamente hasta encontrar la totalidad de los elementos de la grilla $G$ hasta el tiempo $t=0$, lo que corresponde a la solución del problema (figura 2).

\section{Implementación}

La implementación se llevó a cabo mediante la programación en lenguaje Matlab para el método de DFI, ya que es más robusto y sus propiedades de estabilidad son superiores al método explícito [5].

Se realizó una aplicación a un problema presentado por Cortázar [12] evaluando un proyecto de inversión con la opción de realizar o no una inversión al final del año 1, modelada como una opción CALL. Cortázar realiza la evaluación mediante simulación, árbol binomial y $D F$ explícitas $(D F E)$. Los resultados son comparados con la solución analítica propuesta por Black y Scholes [7], logrando para todas las metodologías resultados similares. Se puede concluir que los resultados de la aplicación realizada mediante $D F I$ son consistentes con los anteriores.

El caso real elegido para aplicar la metodología corresponde al proyecto "Implementación de un sistema de información geográfica $(S I G)$ para controlar la cartografía de las plantaciones pertenecientes a Forestal Mininco S.A." estudiado por Becker [6] y resuelto mediante la metodología de "árbol binomial con transformada logarítmica $(A B T L)$ " por Garrido [14].

La metodología $A B T L$ se basa fundamentalmente en considerar que el precio del activo subyacente, que corresponde al valor de los flujos de caja $V$, sigue un proceso estocástico de difusión que puede ser aproximado en tiempo discreto. Así, se subdivide la vida total del proyecto en $N$ subintervalos de tiempo, dentro de los cuales el valor de $X=\ln (V)$ sigue un proceso estocástico Markoviano con movimientos aletorios ascendentes y descendentes, obteniéndose la evolución estocástica del proyecto. Después se calcula iterativamente el valor del proyecto trayendo al presente el valor de los flujos de caja descontados a la tasa libre de riesgo.

Un sistema de información geográfica $(S I G)$ es una colección organizada de hardware, software, datos geográficos y personas, diseñada para capturar, almacenar, actualizar y desplegar eficientemente cualquier información concerniente a la posición, atributos y topología de un determinado terreno. El proyecto de implementación de un $S I G$ para controlar la cartografía de las plantaciones pertenecientes a Forestal Mininco S.A. fue concebido como 
un nuevo sistema de procesamiento de la información que pretendía mejorar y modernizar los métodos de acceso a la información y las herramientas de análisis de los datos geográficos de la empresa. Los beneficios del proyecto se manifestarían en aumentos de eficiencia en el estudio de las alternativas de decisión y ejecución de las acciones operativas que se desprendían de estos análisis. Becker [6] evaluó distintas alternativas para el proyecto, utilizando la metodología de evaluación de decisiones estratégicas propuesta por Del Sol en 1999. Garrido [14] realizó la evaluación de este mismo proyecto mediante la metodología de opciones reales utilizando $A B T L$, considerando las múltiples opciones existentes en el proyecto, obteniendo valores muy contrastantes.

A partir de la información concerniente a los flujos de caja, ingresos, costos, inversiones y opciones de este proyecto, se procede a evaluar el proyecto mediante la metodología de opciones reales aplicando DFI.

Se considera una varianza de los flujos de caja del proyecto de $\sigma^{2}=0.58$ y una tasa libre de riesgo anual de $r=4 \%$. El caso presenta las siguientes opciones:

- Opción de esperar $(E)$ un período hasta $T_{1}=1$ para realizar la inversión inicial $I_{0}=$ US $\$ 201.500$.

- Opción de abandonar $(A)$ no realizando la inversión programada para el año $1, I_{l}=$ US $\$ 135.900$.

- Opción de contraer $(C)$ la escala del proyecto en el año 2 un 30\%, generando un ahorro $I_{c}=$ US\$ 75.000.

- Opción de expandir $(X)$ la escala del proyecto en el año 3 un 20\%, con una inversión $I_{e}=\mathrm{US} \$ 50.000$.

- Opción de cambio $(K)$ a mejor uso alternativo en los años 4 y 5 , considerando un valor de salvamento del proyecto $K=\mathrm{US} \$ 200.000$.

Para el análisis se consideraron 32 casos de opciones y combinaciones de opciones $E, A, C, X$ y $K$. Por ejemplo, se evalúa el proyecto considerando las opciones de contraer $(C)$ en el año 2 y expandir $(X)$ en el año 3, lo que se designa como "alternativa $C+X$ ".

\section{Calibración del método}

Antes de proceder a evaluar el proyecto es imprescindible definir los parámetros asociados a la metodología DFI y que corresponden fundamentalmente al nivel deseado de discretización de tiempo y valores, tanto en el tamaño de los intervalos como en los rangos entre los que se desea que varíen los valores del flujo de caja. Por lo tanto, a modo de calibración se determinó realizar un análisis de sensibilidad para determinar la influencia de estos parámetros en los resultados de la evaluación. En él se variaron la cantidad $M$ de intervalos de valor del flujo de caja y luego el rango de valores máximos y mínimos entre los que podía oscilar el flujo de caja $\left(S_{\max } \mathrm{y} S_{\text {min }}\right)$.

Las diferentes alternativas se evaluaron para el proyecto $S I G$ para las 32 combinaciones de opciones presentadas anteriormente, obteniéndose 32 × $6=192$ tablas de resultados. Los resultados de cada alternativa fueron comparados con los obtenidos mediante $A B T L$ presentados por Garrido (2003) y se presentan de manera resumida en la tabla 1 y figura 3 .

De los resultados obtenidos se observa que la variación en el número de intervalos $M$ (alternativas $D F I 1=20$, DFI $2=50$ y $D F I 3=100)$ prácticamente no altera el resultado, pero sí permite un análisis más detallado de los resultados, ya que al obtenerse una malla más fina (con mayor cantidad de valores e intervalos menores) es más fácil buscar el valor deseado permitiendo la toma de decisiones con mayor facilidad sin ser necesario recurrir a la interpolación.

Sin embargo, se observa que la variación en el rango de valores entre los que puede variar el flujo de caja $S_{\max }$ y $S_{\text {min }}$ (alternativas DFI $4= \pm$ US $\$ 2.000 .000$, DFI $5=$ \pm US\$ 4.000.000 y DFI $6= \pm$ US\$ 8.000.000) influye de manera importante en los resultados que se obtienen. Se puede observar que aumentando el rango de variabilidad del flujo de caja del proyecto de \pm US $\$ 800.000$ a \pm US\$ 8.000.000 (o sea en 10 veces), los valores obtenidos también aumentan en el orden de 10 veces. La razón de este cambio se explica debido a que al aumentar el rango de los valores que puede llegar a tomar el flujo de caja del proyecto, se está aumentando también la incertidumbre del mismo. A mayor incertidumbre que exista, más valiosa será la flexibilidad que pueda presentar el proyecto, por lo que el valor de la opción aumenta. Con estos resultados se grafica de manera muy explícita la importancia que tiene la flexibilidad de un proyecto al enfrentarse a las incertidumbres del mercado.

Revisando los resultados obtenidos de este análisis de sensibilidad se llega a la concusión que si bien los resultados obtenidos mediante $D F I$ difieren de los obtenidos por Garrido [14] mediante $A B T L$, las alternativas DFI 1, DFI 2 y DFI 3 tienen los resultados más parecidos a los del $A B T L$, ya que se mantienen dentro de márgenes similares a diferencia de las otras alternativas que llegan a tener valores demasiado superiores, lo que sirve como calibración del método para el caso a estudiar. 
Tabla 1. Análisis de sensibilidad: VAN tradicional y VAN expandido según resultados obtenidos mediante árbol binomial con transformada logarítmica $(A B T L)$ [14], y mediante diferencias finitas implícitas $(D F I)$ con variación de parámetros $S_{\max }, S_{\min }$ y $M$.

\begin{tabular}{|c|c|c|c|c|c|c|c|c|c|}
\hline & & & & & & Expan & & & \\
\hline Alternativa & Opciones & Tradicional & ABTL & DFI 1 & DFI 2 & DFI 3 & DFI 4 & DFI 5 & DFI 6 \\
\hline 1 & - & 79,6 & 79,6 & 79,6 & 79,4 & 79,4 & 77,9 & 78,1 & 78,5 \\
\hline 2 & $E$ & 79,6 & 281,1 & 79,6 & 79,4 & 79,4 & 77,9 & 78,1 & 78,5 \\
\hline 3 & $\mathrm{~A}$ & 79,6 & 114,1 & 140,5 & 140,0 & 140,0 & 310,0 & 610,4 & 1223,6 \\
\hline 4 & $\mathrm{C}$ & 79,6 & 92,5 & 120,4 & 120,4 & 120,5 & 205,2 & 341,1 & 611,5 \\
\hline 5 & $\mathrm{X}$ & 79,6 & 126,9 & 146,5 & 146,6 & 146,7 & 216,0 & 325,8 & 543,2 \\
\hline 6 & $\mathrm{~K}$ & 79,6 & 121,8 & 247,0 & 246,7 & 246,7 & 659,6 & 1318,9 & 2627,8 \\
\hline 7 & $E+A$ & 79,6 & 315,6 & 140,5 & 140,0 & 140,0 & 310,0 & 610,4 & 1223,6 \\
\hline 8 & $\mathrm{E}+\mathrm{C}$ & 79,6 & 294,0 & 120,4 & 120,4 & 120,5 & 205,2 & 341,1 & 611,5 \\
\hline 9 & $E+X$ & 79,6 & 328,4 & 146,5 & 146,6 & 146,7 & 216,0 & 325,8 & 543,2 \\
\hline 10 & $E+K$ & 79,6 & 323,3 & 247,0 & 246,7 & 246,7 & 659,6 & 1318,9 & 2627,8 \\
\hline 11 & $\mathrm{~A}+\mathrm{C}$ & 79,6 & 127,0 & 154,3 & 153,7 & 153,8 & 343,7 & 668,7 & 1327,7 \\
\hline 12 & $A+X$ & 79,6 & 161,4 & 204,4 & 203,8 & 203,8 & 431,9 & 819,3 & 1602,6 \\
\hline 13 & $\mathrm{~A}+\mathrm{K}$ & 79,6 & 156,3 & 247,0 & 246,7 & 246,7 & 659,6 & 1318,9 & 2627,7 \\
\hline 14 & $\mathrm{C}+\mathrm{X}$ & 79,6 & 139,7 & 186,7 & 186,9 & 187,0 & 340,3 & 582,8 & 1064,0 \\
\hline 15 & $\mathrm{C}+\mathrm{K}$ & 79,6 & 125,5 & 248,6 & 248,1 & 248,1 & 660,8 & 1319,7 & 2628,2 \\
\hline 16 & $\mathrm{X}+\mathrm{K}$ & 79,6 & 170,5 & 317,6 & 317,1 & 317,1 & 813,7 & 1604,7 & 3175,1 \\
\hline 17 & $\mathrm{E}+\mathrm{A}+\mathrm{C}$ & 79,6 & 328,5 & 154,3 & 153,7 & 153,8 & 343,7 & 668,7 & 1327,7 \\
\hline 18 & $E+A+X$ & 79,6 & 362,9 & 204,4 & 203,8 & 203,8 & 431,9 & 819,3 & 1602,6 \\
\hline 19 & $\mathrm{E}+\mathrm{A}+\mathrm{K}$ & 79,6 & 357,8 & 247,2 & 246,7 & 246,7 & 659,6 & 1318,9 & 2627,7 \\
\hline 20 & $\mathrm{E}+\mathrm{C}+\mathrm{X}$ & 79,6 & 341,2 & 186,7 & 186,9 & 187,0 & 340,3 & 582,8 & 1064,0 \\
\hline 21 & $\mathrm{E}+\mathrm{C}+\mathrm{K}$ & 79,6 & 327,0 & 248,6 & 248,1 & 248,1 & 660,8 & 1319,7 & 2628,2 \\
\hline 22 & $\mathrm{E}+\mathrm{X}+\mathrm{K}$ & 79,6 & 372,0 & 317,6 & 317,1 & 317,1 & 813,7 & 1604,7 & 3175,1 \\
\hline 23 & $\mathrm{~A}+\mathrm{C}+\mathrm{X}$ & 79,6 & 174,2 & 218,7 & 218,2 & 218,2 & 468,8 & 885,8 & 1724,6 \\
\hline 24 & $\mathrm{~A}+\mathrm{C}+\mathrm{K}$ & 79,6 & 160,0 & 248,6 & 248,1 & 248,1 & 660,8 & 1319,7 & 2628,2 \\
\hline 25 & $\mathrm{~A}+\mathrm{X}+\mathrm{K}$ & 79,6 & 205,0 & 317,6 & 317,1 & 317,1 & 813,7 & 1604,7 & 3175,1 \\
\hline 26 & $\mathrm{C}+\mathrm{X}+\mathrm{K}$ & 79,6 & 174,0 & 318,9 & 318,4 & 318,4 & 814,7 & 1605,5 & 3175,6 \\
\hline 27 & $\mathrm{E}+\mathrm{A}+\mathrm{C}+\mathrm{X}$ & 79,6 & 375,7 & 218,7 & 218,2 & 218,2 & 468,8 & 885,8 & 1724,6 \\
\hline 28 & $\mathrm{E}+\mathrm{A}+\mathrm{C}+\mathrm{K}$ & 79,6 & 361,5 & 248,6 & 248,1 & 248,1 & 660,8 & 1319,7 & 2628,2 \\
\hline 29 & $\mathrm{E}-\mathrm{A}+\mathrm{X}+\mathrm{K}$ & 79,6 & 406,5 & 317,6 & 317,1 & 317,1 & 813,7 & 1604,7 & 3175,1 \\
\hline 30 & $E+C+X+K$ & 796 & 375,5 & 318,9 & 318,4 & 318,4 & 814,7 & 1605,5 & 3175,6 \\
\hline 31 & $\mathrm{~A}+\mathrm{C}+\mathrm{X}+\mathrm{K}$ & 79,6 & 208,5 & 318,9 & 318,4 & 318,4 & 814,7 & 1605,5 & 3175,6 \\
\hline 32 & $\mathrm{E}+\mathrm{A}+\mathrm{C}+\mathrm{X}+\mathrm{K}$ & 79,6 & 410,0 & 318,9 & 318,4 & 318,4 & 814,7 & 1605,5 & 3175,6 \\
\hline
\end{tabular}

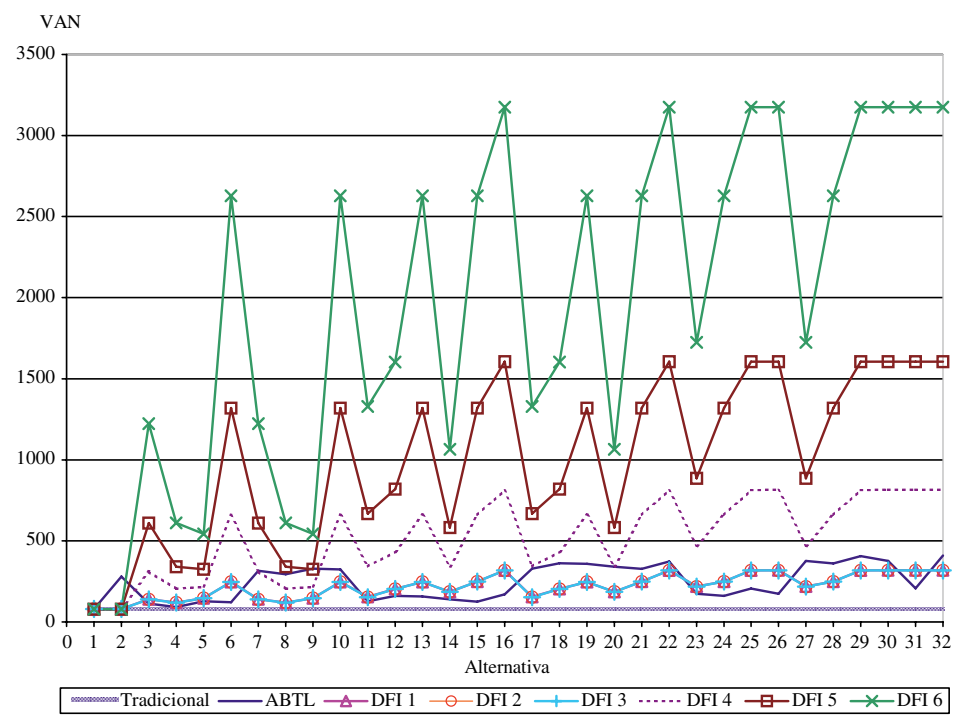

Figura 3. Análisis de sensibilidad: VAN tradicional y VAN expandido según resultados obtenidos mediante árbol binomial con transformada logarítmica $(A B T L)$ [14], y mediante diferencias finitas implícitas $(D F I)$ con variación de parámetros $S_{\max }, S_{\min }$ y $M$. 


\section{Análisis de resultados}

Luego de realizar la evaluación del proyecto $S I G$ mediante la metodología $D F I$ se puede observar lo siguiente:

En la medida en que la opción se encuentra más alejada del inicio $(t=0)$ ésta tiene mayor valor, ya que existe mayor incertidumbre respecto a los comportamientos del mercado y por lo tanto, al encontrarse alejada del inicio del proyecto, la flexibilidad que le entrega al proyecto tiene un mayor valor, lo que se transmite en beneficios económicos más incidentes.

La opción de esperar tiene valor sólo para flujos de caja muy negativos y su valor es muy poco incidente en monto final. Esto se debe a que en un año es muy poca la incertidumbre que existe y por lo tanto el valor de la flexibilidad del proyecto no es tan importante como para situaciones en horizontes más lejanos.

La opción de abandonar ya tiene una influencia significativa en el aumento del $V A N$, incluso mayor al valor obtenido mediante $A B T L$. La opción de abandonar tiene mayor valor para flujos de caja negativos, ya que permite evitar inversiones programadas ante escenarios desfavorables logrando controlar al menos parcialmente las pérdidas.

Al igual que la opción de abandonar, la opción de contraer permite controlar las pérdidas ante flujos de caja desfavorables, lo que se concluye al observar el alto valor que presenta la opción para dichos casos, mientras que para valores altos del flujo de caja el valor de la opción de contraer es cero, lo que resulta lógico. Para la opción de contraer el método $D F I$ también presenta valores del $V A N$ mayores que los obtenidos mediante $A B T L$.

En la medida en que los flujos de caja proyectados aumentan, se incrementa el valor de la opción de expansión, ya que resulta conveniente expandir proyectos ante escenarios favorables, como se puede observar de los resultados obtenidos. Para este caso los resultados obtenidos mediante DFI son del orden de un $15 \%$ mayores que los obtenidos mediante $A B T L$, representando un VAN expandido de un monto correspondiente a casi al doble del VAN tradicional.

La opción de cambio a mejor uso alternativo es en definitiva la opción más incidente en el valor del VAN expandido del proyecto, tanto porque es una opción presente al final del horizonte de evaluación (lo que le da mayor valor a la opción tal como se comentó anteriormente), como por el hecho de que acota fuertemente el rango inferior del flujo de caja. Para esta opción, los resultados mediante DFI son más del doble de los obtenidos mediante $A B T L$, lo que hace contrastar una vez más los resultados de ambas metodologías.

Para todas las combinaciones de opciones presentadas, el $V A N$ del proyecto es superior al obtenido para las opciones consideradas de manera independiente. En la medida en la que existen más opciones presentes en el horizonte de evaluación, aumenta la flexibilidad del proyecto para adaptarse a las condiciones del mercado cuya incertidumbre se va disipando con el paso del tiempo. Esto se traduce en un mayor valor de las opciones presentes y por lo tanto un mayor valor del proyecto. Sin embargo, el VAN obtenido mediante DFI para las 5 opciones actuando simultáneamente es bastante menor al obtenido si se suman las acciones de las 5 opciones analizadas de manera independiente. Esto se debe a que no pueden ser analizadas de manera aislada, ya que existe una interacción entre las distintas opciones influyendo así en los resultados, lo que destaca la importancia de utilizar una metodología que considere dicha interacción de manera de representar de manera correcta la evolución del proyecto sin sobrestimar la influencia de las opciones presentes.

El VAN obtenido mediante DFI para las 5 opciones presentes de manera simultánea es bastante mayor que el obtenido mediante los métodos tradicionales, lo que nos demuestra que el valor de las suma de las opciones reales presentes en el proyecto representa la flexibilidad inherente al proyecto y que le permitirá adaptarse a las cambiantes condiciones del mercado sin seguir un camino rígido preestablecido.

\section{CONCLUSIONES}

Una de las grandes falencias de los métodos tradicionales de evaluación de proyectos corresponde a la incapacidad de representar adecuadamente el valor asociado a la flexibilidad operativa que posee cada proyecto. La flexibilidad de un proyecto representa su capacidad de adaptarse a las condiciones inciertas en las que evoluciona el mercado, tanto para condiciones favorables (maximizando los ingresos) como para condiciones desfavorables (eliminando parcial o totalmente las pérdidas, según sea el caso). De esta manera el proyecto podrá tomar distintos caminos de expansión, contracción, aplazamiento de inversiones, receso temporal o por último el abandono definitivo, sin estar basado en una política rígida de decisiones tomadas al inicio del proyecto.

La evaluación de proyectos debe por lo tanto considerar la flexibilidad de un proyecto dentro del análisis, ya 
que representa una parte muy importante del valor real de un proyecto. Es así como nace la teoría de opciones reales, basada en la teoría financiera y que tiene como fin capturar numéricamente el valor de la flexibilidad de un proyecto. Esto implica que en ocasiones, proyectos evaluados mediante las metodologías tradicionales podrían parecer no rentables, pero mediante la teoría de opciones reales el proyecto puede resultar conveniente y por lo tanto puede llevarse a cabo. Así, proyectos que tengan más alternativas y mayor flexibilidad para adaptarse ante condiciones cambiantes, van a tener un mayor valor al momento de ser evaluados adecuadamente.

Por lo tanto, la incertidumbre pasa a ser un elemento que le entrega valor al proyecto, ya que ante escenarios de mayor incertidumbre, la flexibilidad operativa del proyecto cobra mayor importancia, de manera que al incorporarla en el análisis el proyecto tendrá un mayor valor.

Las opciones reales presentes en un proyecto tendrán mayor incidencia en la medida en que se presenten hacia el fin del horizonte de evaluación y menos en la medida en que se presenten cerca del origen del proyecto. Así también cuando existen distintas opciones presentes en un horizonte de evaluación es indispensable analizarlas de manera conjunta, ya que existirá una interacción entre ellas que debe ser considerada.

Las opciones reales incorporan el concepto de evaluación bajo la inexistencia de oportunidades de arbitraje y permiten lograr el alineamiento entre las decisiones estratégicas de inversión y los mercados financieros. Esto se realiza mediante la utilización de un activo subyacente transado en el mercado, con una rentabilidad esperada igual a la del proyecto, que represente a través de la evolución de su precio en el tiempo, el comportamiento de la variabilidad de los flujos de caja del proyecto durante el horizonte de evaluación. Una de las ventajas más importantes de esta metodología es que proporciona una política óptima de operación que maximiza el valor del activo real. Tal política se caracteriza por entregar un conjunto de valores críticos de las variables inciertas presentes en un proyecto, que indica cuándo y en qué instante es óptimo ejercitar la opción.

Cuando se utiliza la teoría de opciones reales para la evaluación de instrumentos derivados, comúnmente se debe resolver una ecuación diferencial parcial. En el caso de activos simples esta ecuación cuenta con una solución analítica. Sin embargo, si la evaluación del activo es más complicada o incorpora más de una opción, por lo general no existe una fórmula exacta para su resolución, ya que la ecuación diferencial parcial tiene tantas dimensiones como variables de estado se usen en la evaluación. Por consiguiente, frente a estas condiciones se hace necesario recurrir a métodos numéricos, los cuales gracias a los avances computacionales han permitido dar solución a problemas de gran dificultad.

Los métodos de integración numérica y los esquemas de $D F E$ y $D F I$ aproximan la ecuación diferencial parcial que se obtiene y valorizan un derivado resolviendo la ecuación diferencial que es satisfecha por el derivado. La ecuación diferencial es convertida en una serie de ecuaciones diferenciales, las que son resueltas de manera iterativa. Pertenece a la categoría de técnicas de evaluación backward-moving, es decir, se comienza en la fecha de expiración del activo que se está evaluando, trayendo los valores que se obtienen hasta el presente. Una de las grandes ventajas de este método corresponde a la riqueza en teoría, algoritmos y avances computacionales existentes que pueden ser utilizados para enfrentar el problema. Presenta además buena convergencia y estabilidad y ofrece una considerable flexibilidad en las opciones de mallas en las dimensiones de tiempo y espacio, resultando útil al momento de analizar los resultados obtenidos ante diversas condiciones del entorno.

El método DFI implementado mediante el programa Matlab fue aplicado a un caso obtenido de la literatura y al comprobar su correcto desempeño se realizó la evaluación económica del proyecto "Implementación de un sistema de información geográfica $(S I G)$ para controlar la cartografía de las plantaciones pertenecientes a Forestal Mininco S.A." estudiado previamente por Becker [6] y evaluado mediante la metodología de "Árbol binomial con transformada logarítmica (ABTL)" por Garrido [14]. El proyecto cuenta con las opciones de esperar, abandonar, contraer, expandir y cambiar a mejor uso alternativo, y fue evaluado para todas las opciones y combinaciones de opciones posibles. Los resultados fueron comparados con la metodología tradicional $(V A N)$ y con los resultados obtenidos por Garrido [14]. Las dos metodologías (DFI y $A B T L)$ entregan valores equivalentes, siendo ambos superiores a los obtenidos mediante la metodología tradicional $(V A N)$, diferencia que representa su capacidad de incorporar en el análisis el valor de la flexibilidad operativa del proyecto.

La teoría de opciones reales para la evaluación de proyectos en conjunto con la metodología de diferencias finitas representa una herramienta de gran utilidad para evaluar proyectos ante escenarios de incertidumbre, permitiendo reflejar la flexibilidad operativa del proyecto de manera numérica y facilitando la decisión de invertir en proyectos que puedan tener escaso valor económico pero alto valor estratégico. 


\section{REFERENCIAS}

[1] P. Acosta. "Modelo de simulación de Montecarlo para evaluar opciones reales: evaluación de una mina de cobre mediante el algoritmo de Barraquad - Martineau". Tesis para optar al grado de magíster en ciencias de ingeniería. Pontificia Universidad Católica de Chile. Santiago, Chile. 1999.

[2] R. Adner and D. Levinthal. "What is not a real option: Considering boundaries for the application of real options to business strategy". Academy of Management Review. Vol. 29 No 1, pp. 74-85. 2004

[3] M. Amram y N. Kulatilaka. "Con mucha disciplina". Estrategia. Vol. $1 \mathrm{~N}^{\circ}$ 4, pp. 40-50. 1999.

[4] M. Amram y N. Kulatilaka. "Opciones reales: Evaluación de inversiones en el mundo incierto". Gestión 2000. Primera Edición. 2000.

[5] E. Arrilucea. "Valoración de un proyecto de edificación mediante opciones reales". Universidad del País Vasco. España. 2004.

[6] J. Becker. "Tecnologías de Información estratégicas: Análisis y aplicación en una empresa forestal". Informe de Tesis. Universidad de Concepción. Concepción, Chile. 2001.

[7] F. Black and M. Scholes. "The pricing of options and corporate liabilities". The Journal of Economy. Vol. 81, pp. 637-654. 1973.

[8] M. Brennan and E. Schwartz. "Evaluating natural resource investments". The Journal of Business. Vol. 58, pp. 135-157. 1985.

[9] M. Broadie and J. Detemple. "Option pricing: Valuation models and applications". Management Science. Vol. 50, pp. 1145-1177. 2004.

[10] T. Copeland, T. Koller and J. Murrin. "Valuation: Measuring and managing the value of companies". John Wiley \& Sons. 2001.

[11] G. Cortázar, M. Gravet and M.J. Urzua. "The valuation of multidimentional american real options using computer based simulation". Pontificia Universidad Católica de Chile. 2004.
[12] G. Cortázar. "Simulation and numerical methods in real options valuation". Pontificia Universidad Católica de Chile. 2001.

[13] A. Dixit and R. Pindyck. "Investment under uncertainty". Princeton University Press, pp. 468. 1995.

[14] I. Garrido. "Análisis y estudio de metodología de opciones reales para la evaluación comparada de proyectos de inversión". Tesis para optar al título de ingeniero civil industrial. Universidad de Concepción. Concepción, Chile. 2003.

[15] M. Gravet. "Valoración de opciones reales mediante simulación: El método de los mínimos cuadrados". Tesis para optar al grado de magíster. Pontificia Universidad Católica de Chile. 2003.

[16] S. Fredes. "Leyes de corte óptimas y valor de una mina con reservas finitas utilizando la teoría de opciones reales". Tesis para optar al grado de magíster. Pontificia Universidad Católica de Chile. 1998.

[17] J. Hull. "Options, futures, and other derivatives". Prentice Hall. 5th Edition, pp. 572. 2002.

[18] T. Luhermann. "Investment opportunities as real options: Getting started on the numbers". Harvard Business Review. N ${ }^{\circ} 76$, pp. 51-67. July - August 1998.

[19] T. Luhermann. "What's it worth?". Harvard Business Review, pp. 132-142. May-June 1997.

[20] E. Schwarz and L. Trigeorgis. "Real options and investment under uncertainty: Classical readings and recent contributions". MIT Press. Chapter 27. 2001.

[21] D. Tavella and K. Randall. "Pricing financial instruments: The finite difference method". John Wiley \& Sons. Inc, pp. 236. 2000.

[22] L. Trigeorgis. "Real options: Managerial flexibility and strategy in resource allocation". MIT Press, pp. 427. 1996.

[23] T. Wang. "Real options "in" projects and systems design: identification of options and solutions for path dependency". Thesis to obtain the degree of doctor. Massachusetts Institute of Technology. Engineering System Division 2005. 